\title{
Potential spaces on fractals
}

\author{
by \\ JiAXin Hu (Beijing) and Martina ZäHLE (JENA)
}

\begin{abstract}
We introduce potential spaces on fractal metric spaces, investigate their embedding theorems, and derive various Besov spaces. Our starting point is that there exists a local, stochastically complete heat kernel satisfying a two-sided estimate on the fractal considered.
\end{abstract}

1. Introduction. The classical Besov spaces $B_{p q}^{s}\left(\mathbb{R}^{n}\right)$ with $s>0$ and $1 \leq p, q \leq \infty$ are closely related to the Gauss-Weierstrass and the CauchyPoisson semigroups. This goes back to Taibleson [25] and Flett [10]. Similarly, the fractional Sobolev spaces $H_{p}^{s}\left(\mathbb{R}^{n}\right), s \in \mathbb{R}, 1 \leq p \leq \infty$, may be expressed in terms of these semigroups. In particular, for $s>0$ they can be interpreted as potential spaces (see for example [22, Chapter V]). The corresponding Gauss-Weierstrass and Cauchy-Poisson heat kernels are explicitly given on $\mathbb{R}^{n}$.

For several fractal sets, local Dirichlet forms and sub-Gaussian heat kernel estimates of the corresponding semigroups have been obtained. A new and interesting phenomenon has been discovered that the walk dimensions of the heat kernels on these fractal sets are strictly greater than 2. The importance of the walk dimension $w$ is that the number $w / 2$ measures the smoothness degree of functions defined on the underlying space. As to the sub-Gaussian estimates of the heat kernels, the reader may refer to the pioneering paper [4] for the Sierpiński gaskets, to [3] for the Sierpiński carpets, [9] for nested fractals, and to [13, 18] for a certain class of post-critically finite fractals. The associated Markov processes are Brownian motions on the fractal sets.

In the present paper the setting is a complete metric space $(X, \varrho)$ admitting a heat kernel with respect to a Radon measure $\mu$ supported on $X$.

2000 Mathematics Subject Classification: Primary 31C28; Secondary 46E58.

Key words and phrases: potential space, Besov space, Bessel (Riesz) potential, heat kernel, fractal.

JH was supported by NSFC (Grant No. 10371062) and the Alexander von Humboldt Foundation. 
Our main assumption is that the heat kernel is local and satisfies two-sided decay estimates (see (G6) and (G7) below). By means of the associated semigroup $\left\{G_{t}\right\}_{t \geq 0}$ we introduce function spaces on $X$ which agree with those mentioned above for $X=\mathbb{R}^{n}$. As in the Euclidean case we obtain some characterizations and embedding theorems. (This may be applied for nonlinear PDE's on $X$.) The opposite way is to introduce function spaces on fractals in a more direct way (see for example $[17,26,27,23]$ ) and to find heat kernel estimates related to associated $p$-energy forms. First steps in this direction are [7] for some non-local structure and [29] for the local version on $d$-sets, when $p=2$ and the walk dimension is equal to 2 .

The paper is organized as follows: in Section 2 we first introduce potential operators of a general strongly continuous contractive semigroup $\left\{G_{t}\right\}_{t \geq 0}$ in the same way as for the Gauss-Weierstrass semigroup. They coincide with the positive fractional powers of the operators $\left(I-A_{p}\right)^{-1}$ and $\left(-A_{p}\right)^{-1}$ (provided they exist), respectively, where $A_{p}$ denotes the $p$-generator of $\left\{G_{t}\right\}_{t \geq 0}$. Then we obtain a formula for the positive fractional powers of $I-A_{p}$ as inverse operators. (The proof is similar to the case of Gauss-Weierstrass semigroups [20].) If $\left\{G_{t}\right\}_{t \geq 0}$ has a local heat kernel satisfying some two-sided decay estimates, the generator $A_{p}$ may be interpreted as a $p$-Laplacian. Under an integrability condition for the upper bound, the reference measure $\mu$ is shown to be a $d$-measure. Moreover, the potential operators of order $\alpha$ in this case have integral kernels (locally) equivalent to $\varrho(x, y)^{d-\alpha w / 2}$, which corresponds to the classical case if $d=n$ and $w=2$. Thus we may interpret these operators as the $p$-Bessel and $p$-Riesz potential operators.

In Section 3 fractional Sobolev spaces on $X$ are introduced as the Bessel potential spaces for local heat kernels satisfying the decay condition. For the special case $p=2$ and $0<\alpha \leq 1$, we obtain characterizations which lead, for $d$-subsets in $\mathbb{R}^{n}$, to the well known Besov spaces introduced in [17] with the method of traces for $w=2,0<\alpha<1$, and to the Lipschitz spaces initiated in [16] for $w \geq 2, \alpha=1$.

In Section 4 embeddings of Sobolev spaces in $L_{q}(\mu)$ spaces or in Hölder spaces are discussed. The classical cases are again included.

Finally, Section 5 complements these contributions by the definition of Besov spaces $B_{p q}^{\alpha}(\mu)$ related to the semigroup $\left\{G_{t}\right\}_{t \geq 0}$. For $p=q=2$, they coincide with the corresponding Sobolev spaces, which generalizes the Euclidean case.

\section{Bessel and Riesz potentials}

2.1. Assumptions. Let $(X, \varrho)$ be a separable, complete metric space that is generally interpreted as a bounded or unbounded fractal. Let $\mu$ be a locally finite Borel measure with $\operatorname{supp} \mu=X$. Assume that $(X, \varrho)$ is connected in 
the sense that $(X, \varrho)$ satisfies the chain condition, that is, there exists a constant $C>0$ such that, for any two points $x, y \in X$ and for any positive integer $n$, there is a sequence $\left\{x_{i}\right\}_{i=0}^{n}$ of points in $X$ with $x_{0}=x, x_{n}=y$, and

$$
\varrho\left(x_{i}, x_{i+1}\right) \leq C n^{-1} \varrho(x, y), \quad 0 \leq i \leq n-1 .
$$

Let $G(t, x, y)$ be a stochastically complete heat kernel or transition density on $(X, \varrho, \mu)$, that is, $G(t, x, y)$ is a real-valued function on $(0, \infty) \times X \times X$ satisfying the following conditions: for all $0<t<\infty$ and $x, y \in X$,

(G1) (non-negativity): $G(t, x, y) \geq 0$;

(G2) (symmetry): $G(t, x, y)=G(t, y, x)$;

(G3) (semigroup property):

$$
G(s+t, x, y)=\int_{X} G(s, x, z) G(t, z, y) d \mu(z) \quad(s>0)
$$

(G4) (identity approximation): $\lim _{t \rightarrow 0+}\left\|G_{t} f-f\right\|_{p}=0$ for any $f \in L_{p}(\mu)$ where $1 \leq p<\infty$, and $\lim _{t \rightarrow 0+} G_{t} f(x)=f(x)$ for $\mu$-almost all $x \in X$ if $f \in L_{\infty}(\mu)$;

(G5) (stochastic completeness): $\int_{X} G(t, x, y) d \mu(y)=1$.

Here $L_{p}(\mu):=L_{p}(X, \varrho, \mu)$ is the usual space of real-valued $p$-integrable functions with norm

$$
\|f\|_{p}:=\left(\int_{X}|f(x)|^{p} d \mu(x)\right)^{1 / p} \quad(1 \leq p<\infty), \quad\|f\|_{\infty}:=\underset{x \in X}{\operatorname{ess} \sup }|f(x)| .
$$

Furthermore $\left\{G_{t}\right\}_{t \geq 0}$ is the semigroup associated with $G(t, x, y)$ :

$$
G_{t} f(x)=\int_{X} G(t, x, y) f(y) d \mu(y) \quad(t>0, x \in X) .
$$

(As usual we set $G_{0}=I$, the identity operator on $L_{p}(\mu)$.)

Remark. Condition (G5) is important in this paper, and it cannot be replaced by a weaker version

$$
\int_{X} G(t, x, y) d \mu(y) \leq 1 \quad(t>0, x \in X) .
$$

EXAMPle 2.1 (Classical case). Let $X=\mathbb{R}^{n}$ with $\varrho$ the Euclidean metric and $\mu$ be the Lebesgue measure. It is easy to see that the Gauss-Weierstrass heat kernel

$$
G_{\mathbb{R}^{n}}(t, x, y)=\left(\frac{1}{4 \pi t}\right)^{n / 2} \exp \left(-\frac{|x-y|^{2}}{4 t}\right)
$$

satisfies (G1)-(G5). The Cauchy-Poisson heat kernel

$$
\begin{aligned}
& P_{\mathbb{R}^{n}}(t, x, y)=C_{n} t^{-n}\left(1+t^{-2}|x-y|^{2}\right)^{-(n+1) / 2} \quad\left(C_{n}=\pi^{-(n+1) / 2} \Gamma((n+1) / 2)\right) \\
& \text { also satisfies (G1)-(G5). }
\end{aligned}
$$


EXAMPLE 2.2 (Fractal case).

1 (Brownian motion on the Sierpinski carpet). Let $X$ be a Sierpiński carpet in $\mathbb{R}^{n}$ with the Euclidean metric, and let $\mu$ be the $d$-dimensional Hausdorff measure on $X$. Barlow and Bass [3] showed that there exists a heat kernel on $X$ satisfying

$$
\begin{aligned}
& a_{1} t^{-d / w} \exp \left(-b_{1}\left(t^{-1 / w}|x-y|\right)^{\gamma}\right) \\
& \leq G(t, x, y) \leq a_{2} t^{-d / w} \exp \left(-b_{2}\left(t^{-1 / w}|x-y|\right)^{\gamma}\right)
\end{aligned}
$$

for all $x, y \in X$ and all $0<t<\operatorname{diam}(X)$, where $w>2$ and $\gamma=w /(w-1)$, $a_{i}, b_{i}>0(i=1,2)$. The Barlow-Bass heat kernel satisfies (G1)-(G5).

2 (Stable-like processes). For each $0<\sigma<1$, there is a function $p^{(\sigma)}(t, x, y)$ on a Sierpiński carpet in $\mathbb{R}^{n}$ satisfying (G1)-(G5) and

$$
\begin{aligned}
& a_{1} t^{-d / \sigma w}\left(1+t^{-1 / \sigma w}|x-y|\right)^{-(d+\sigma w)} \\
& \quad \leq p^{(\sigma)}(t, x, y) \leq a_{2} t^{-d / \sigma w}\left(1+t^{-1 / \sigma w}|x-y|\right)^{-(d+\sigma w)}
\end{aligned}
$$

for all $x, y \in X$ and all $0<t<\operatorname{diam}(X)$, where $a_{1}, a_{2}>0$ (see for example [5] or [15]).

Notation. Throughout this paper we denote by $C$ a general constant and let $r_{0}=\operatorname{diam}(X) \in(0, \infty]$. Two non-negative functions $f$ and $g$ are equivalent, denoted by $f \cong g$, if $C^{-1} f(x) \leq g(x) \leq C f(x)$ for all $x \in X$ and some $C>0$. By the "classical case" we mean $X=\mathbb{R}^{n}, \varrho(x, y)=|x-y|$ and $\mu$ is the Lebesgue measure on $\mathbb{R}^{n}$ (see Example 2.1).

By (G3) and (G4), we see that $\left\{G_{t}\right\}_{t \geq 0}$ is strongly continuous on $L_{p}(\mu)$ $(1 \leq p<\infty)$ :

$$
\lim _{t \rightarrow t_{0}}\left\|G_{t} f-G_{t_{0}} f\right\|_{p}=0 \quad\left(f \in L_{p}(\mu), t_{0} \geq 0\right),
$$

and by (G5), $\left\{G_{t}\right\}_{t \geq 0}$ is contractive:

$$
\left\|G_{t} f\right\|_{p} \leq\|f\|_{p} \quad\left(t \geq 0, f \in L_{p}(\mu), 1 \leq p \leq \infty\right) .
$$

Therefore, there exists an infinitesimal generator $A_{p}$ of $\left\{G_{t}\right\}_{t \geq 0}$ on $L_{p}(\mu)$ $(1 \leq p<\infty)$ :

$$
A_{p} f=\lim _{t \rightarrow 0+} \frac{G_{t} f-f}{t} \quad \text { strongly in } L_{p}(\mu)
$$

with domain $\mathcal{D}\left(A_{p}\right)$, the space of all functions $f$ such that the limit in (2.5) exists. Note that $\mathcal{D}\left(A_{p}\right)$ is dense in $L_{p}(\mu)$ (see for example [28, p. 237]).

In the following we will repeatedly specify to $L_{2}(\mu)$. For simplicity we write $A:=A_{2}$ and $\mathcal{D}(A):=\mathcal{D}\left(A_{2}\right)$. By $(\mathrm{G} 2)$, we see that $A$ is self-adjoint:

$$
(A f, g)=(f, A g) \quad(f, g \in \mathcal{D}(A)),
$$


where $($,$) is the inner product in L_{2}(\mu)$,

$$
(f, g):=\int_{X} f(x) g(x) d \mu(x) .
$$

Moreover, the linear operator $A$ is non-positive definite:

$$
(A f, f) \leq 0 \quad(f \in \mathcal{D}(A))
$$

since, by (G5) and (G1),

$$
(A f, f)=-\lim _{t \rightarrow 0} \mathcal{E}_{t}(f, f) \leq 0 \quad(f \in \mathcal{D}(A)),
$$

where

$$
\mathcal{E}_{t}(f, g):=\frac{1}{2 t} \int_{X} \int_{X}(f(y)-f(x))(g(y)-g(x)) G(t, x, y) d \mu(y) d \mu(x) .
$$

Thus $-A$ admits a unique spectral resolution:

$$
-A f=\int_{0}^{\infty} \lambda d E_{\lambda} f \quad(f \in \mathcal{D}(A))
$$

(see for example [28, p. 313]). Note that

$$
G_{t} f=\int_{0}^{\infty} e^{-\lambda t} d E_{\lambda} f \quad\left(f \in L_{2}(\mu), t \geq 0\right) .
$$

For $\alpha \in \mathbb{R}$, we define

$$
\begin{aligned}
\mathcal{D}\left((-A)^{\alpha}\right) & =\left\{f \in L_{2}(\mu): \int_{0}^{\infty} \lambda^{2 \alpha} d\left(E_{\lambda} f, f\right)<\infty\right\}, \\
(-A)^{\alpha} f & =\int_{0}^{\infty} \lambda^{\alpha} d E_{\lambda} f \quad\left(f \in \mathcal{D}\left((-A)^{\alpha}\right)\right) .
\end{aligned}
$$

The unique Dirichlet form $(\mathcal{E}, \mathcal{F})$ associated with $A$ is determined by

$$
\begin{aligned}
& \mathcal{F}=\mathcal{D}\left((-A)^{1 / 2}\right), \\
& \mathcal{E}(f, g)=\left((-A)^{1 / 2} f,(-A)^{1 / 2} g\right) \quad(f, g \in \mathcal{F})
\end{aligned}
$$

(see for example [11]). We may characterize $(\mathcal{E}, \mathcal{F})$ in terms of $G(t, x, y)$ as follows:

$$
\begin{aligned}
& \mathcal{F}=\left\{f \in L_{2}(\mu): \lim _{t \rightarrow 0} \mathcal{E}_{t}(f, f)<\infty\right\}, \\
& \mathcal{E}(f, g)=\lim _{t \rightarrow 0} \mathcal{E}_{t}(f, g) \quad(f, g \in \mathcal{F}) .
\end{aligned}
$$

Note that (G5) plays an important part in obtaining (2.13).

2.2. Potentials associated with semigroups. In what follows we will study two distinct kinds of potentials associated with semigroups as above. We first consider the general case of a strongly continuous contractive semigroup 
$\left\{G_{t}\right\}_{t \geq 0}$ on the Banach space $L_{p}(\mu), p \geq 1$ (where the kernel assumption in Subsection 2.1 is not needed).

Definition 2.3. For $\alpha>0$ and $1 \leq p \leq \infty$, the potential operators of order $\alpha$ of $f \in L_{p}(\mu)$ are defined by

$$
\begin{aligned}
& J_{\mu}^{\alpha} f(x)=\frac{1}{\Gamma(\alpha / 2)} \int_{0}^{\infty} t^{\alpha / 2-1} e^{-t} G_{t} f(x) d t \\
& I_{\mu}^{\alpha} f(x)=\frac{1}{\Gamma(\alpha / 2)} \int_{0}^{\infty} t^{\alpha / 2-1} G_{t} f(x) d t \quad(x \in X) .
\end{aligned}
$$

Remarks. 1. Note that $I_{\mu}^{\alpha} f$ may not be well defined for some $\alpha$ and $f$, if $X$ is bounded. For example, if $f=1$ then $I_{\mu}^{\alpha} f=\infty$ for any $\alpha>0$. However, $J_{\mu}^{\alpha} f$ is well defined for any $\alpha>0$ and any $f \in L_{p}(\mu), 1 \leq p \leq \infty$, because $J_{\mu}^{\alpha} f \in L_{p}(\mu)$ for $f \in L_{p}(\mu)$ due to the fact that $\left\|J_{\mu}^{\alpha} f\right\|_{p} \leq\|f\|_{p}$. For this reason we will mostly work with the potential operators $J_{\mu}^{\alpha}$.

2. For the classical case, if $G(t, x, y)$ is the Gauss-Weierstrass heat kernel, then $J_{\mu}^{\alpha}$ and $I_{\mu}^{\alpha}$ defined in Definition 2.3 are the Bessel and Riesz potential operators respectively (see for example [22] or [20, 21]). For the Sierpiński carpet $X$, in order that $J_{\mu}^{\alpha}$ and $I_{\mu}^{\alpha}$ be Bessel and Riesz potential operators, we take for $G(t, x, y)$ the Barlow-Bass heat kernel (see Subsection 2.3 below).

The operator $J_{\mu}^{\alpha}$ or $I_{\mu}^{\alpha}$ in Definition 2.3 may be interpreted as the fractional power of order $\alpha / 2$ of the resolvent $\left(I-A_{p}\right)^{-1}$ or of $\left(-A_{p}\right)^{-1}$ (should it exist) by the following arguments. We first consider the case $p=2$ with symmetric operators $G_{t}$, where such powers are introduced by means of the spectral resolution.

Proposition 2.4. Let $J_{\mu}^{\alpha}$ be defined as in (2.14), and let $A$ be the generator of a symmetric strongly continuous contractive semigroup $\left\{G_{t}\right\}_{t \geq 0}$ on $L_{2}(\mu)$. Then the following holds on $L_{2}(\mu)$ :

$$
J_{\mu}^{\alpha}=(I-A)^{-\alpha / 2} \quad(\alpha>0) .
$$

Proof. Let $\alpha>0$. By (2.10) and Fubini's theorem, we have

$$
\begin{aligned}
\frac{1}{\Gamma(\alpha / 2)} \int_{0}^{\infty} t^{\alpha / 2-1} e^{-t} G_{t} f d t & =\frac{1}{\Gamma(\alpha / 2)} \int_{0}^{\infty} t^{\alpha / 2-1} e^{-t}\left(\int_{0}^{\infty} e^{-\lambda t} d E_{\lambda} f\right) d t \\
& =\frac{1}{\Gamma(\alpha / 2)} \int_{0}^{\infty}\left(\int_{0}^{\infty} t^{\alpha / 2-1} e^{-(1+\lambda) t} d t\right) d E_{\lambda} f \\
& =\int_{0}^{\infty}(1+\lambda)^{-\alpha / 2} d E_{\lambda} f=(I-A)^{-\alpha / 2} f, \quad f \in L_{2}(\mu),
\end{aligned}
$$

which combines with (2.14) to yield (2.16). 
For general $1 \leq p \leq \infty$, we may use the notation

$$
\left(I-A_{p}\right)^{-\alpha / 2}:=J_{\mu}^{\alpha},
$$

since $\left(I-A_{p}\right)^{-1}=J_{\mu}^{2}$, and

$$
J_{\mu}^{\alpha_{1}+\alpha_{2}}=J_{\mu}^{\alpha_{1}} \circ J_{\mu}^{\alpha_{2}} \quad\left(\alpha_{1}, \alpha_{2}>0\right) .
$$

The last equality follows from (2.14) and the semigroup property of $\left\{G_{t}\right\}_{t \geq 0}$. One can also use the notation

$$
\left(-A_{p}\right)^{-\alpha / 2}:=I_{\mu}^{\alpha}
$$

by similar arguments for suitable $\alpha$ and $f$.

Note that (2.16) may formally be obtained as follows. Since

$$
(1+u)^{-\alpha / 2}=\frac{1}{\Gamma(\alpha / 2)} \int_{0}^{\infty} t^{\alpha / 2-1} e^{-(1+u) t} d t \quad(\alpha>0)
$$

we replace $u$ by $-A$ and then use $G_{t}=e^{A t}$ to obtain

$$
(I-A)^{-\alpha / 2}=\frac{1}{\Gamma(\alpha / 2)} \int_{0}^{\infty} t^{\alpha / 2-1} e^{-t} e^{A t} d t=\frac{1}{\Gamma(\alpha / 2)} \int_{0}^{\infty} t^{\alpha / 2-1} e^{-t} G_{t} d t=J_{\mu}^{\alpha} .
$$

Similarly, we replace $1+u$ by $u$ in (2.17), and then let $u=-A$ to obtain

$$
(-A)^{-\alpha / 2}=\frac{1}{\Gamma(\alpha / 2)} \int_{0}^{\infty} t^{\alpha / 2-1} e^{A t} d t=\frac{1}{\Gamma(\alpha / 2)} \int_{0}^{\infty} t^{\alpha / 2-1} G_{t} d t=I_{\mu}^{\alpha} .
$$

We will see that there exists an explicit formula for the inversion of $J_{\mu}^{\alpha}$ for any $\alpha>0$ and $1 \leq p \leq \infty$, as in the classical case (cf. [20, 21]). For this, define

$$
D_{\varepsilon}^{\alpha}=\frac{1}{\chi(\alpha / 2, l)} \int_{\varepsilon}^{\infty} t^{-\alpha / 2-1}\left(I-e^{-t} G_{t}\right)^{l} d t \quad(\varepsilon>0)
$$

for $\alpha>0$, where $l=[\alpha / 2]+1([\alpha / 2]$ is the integer part of $\alpha / 2)$ and

$$
\chi(\alpha / 2, l)=\int_{0}^{\infty} s^{-\alpha / 2-1}\left(1-e^{-s}\right)^{l} d s<\infty \quad(0<\alpha / 2<l) .
$$

THEOREM 2.5. Let $\left\{G_{t}\right\}_{t \geq 0}$ be a strongly continuous contractive semigroup on $L_{p}(\mu)$ and $\alpha>0$. If $1 \leq p<\infty$, the left inverse of $J_{\mu}^{\alpha}$ exists in the following sense:

$$
\lim _{\varepsilon \rightarrow 0}\left\|D_{\varepsilon}^{\alpha} J_{\mu}^{\alpha} f-f\right\|_{p}=0
$$

for any $f \in L_{p}(\mu)$. Moreover, if $p=\infty$ then

$$
\lim _{\varepsilon \rightarrow 0} D_{\varepsilon}^{\alpha} J_{\mu}^{\alpha} f(x)=f(x)
$$

for $\mu$-almost all $x \in X$ and $f \in L_{\infty}(\mu)$. 
Proof. The proof is similar to the classical case (see for example [20, Theorem 20.4, pp. 260-261] or [1, Theorem 1]). For the reader's convenience, we sketch the proof. Let $f \in L_{p}(\mu)(1 \leq p \leq \infty)$. By (2.18), we see that

$$
D_{\varepsilon}^{\alpha} J_{\mu}^{\alpha} f=\frac{1}{\chi(\alpha / 2, l)} \int_{\varepsilon}^{\infty} t^{-\alpha / 2-1}\left\{\sum_{k=0}^{l}(-1)^{k}\left(\begin{array}{l}
l \\
k
\end{array}\right) e^{-k t} G_{k t}\left(J_{\mu}^{\alpha} f\right)\right\} d t .
$$

By (2.14) and the semigroup property,

$$
\begin{aligned}
e^{-k t} G_{k t}\left(J_{\mu}^{\alpha} f\right) & =\frac{1}{\Gamma(\alpha / 2)} \int_{0}^{\infty} s^{\alpha / 2-1} e^{-(s+k t)} G_{s+k t} f d s \\
& =\frac{1}{\Gamma(\alpha / 2)} \int_{0}^{\infty}\left((s-k t)_{+}\right)^{\alpha / 2-1} e^{-s} G_{s} f d s
\end{aligned}
$$

where

$$
a_{+}= \begin{cases}a, & a \geq 0 \\ 0, & \text { otherwise. }\end{cases}
$$

Thus, it follows from (2.22) that

$$
D_{\varepsilon}^{\alpha} J_{\mu}^{\alpha} f=\frac{1}{\chi(\alpha / 2, l) \Gamma(\alpha / 2)} \int_{0}^{\infty} e^{-s} G_{s} f\left\{\sum_{k=0}^{l}(-1)^{k}\left(\begin{array}{l}
l \\
k
\end{array}\right) \psi_{k, \varepsilon}(s)\right\} d s
$$

where

$$
\psi_{k, \varepsilon}(s):=\int_{\varepsilon}^{\infty} t^{-\alpha / 2-1}\left((s-k t)_{+}\right)^{\alpha / 2-1} d t .
$$

It is not hard to calculate that

$$
\psi_{k, \varepsilon}(\varepsilon s)=\frac{2}{\alpha \varepsilon s}\left((s-k)_{+}\right)^{\alpha / 2} \quad(s, k \geq 0) .
$$

Therefore, we see from (2.23) that

$$
D_{\varepsilon}^{\alpha} J_{\mu}^{\alpha} f=\int_{0}^{\infty} K_{\alpha / 2, l}(s) e^{-\varepsilon s} G_{\varepsilon s} f d s
$$

where

$$
K_{\beta, l}(s)=\frac{1}{\chi(\beta, l) \Gamma(\beta+1)} \sum_{k=0}^{l}(-1)^{k}\left(\begin{array}{l}
l \\
k
\end{array}\right) s^{-1}\left((s-k)_{+}\right)^{\beta}
$$

for any $\beta>0$ with $l=[\beta]+1$. The function $K_{\beta, l}$ has the following properties: $K_{\beta, l} \in L_{1}(0, \infty)$, and

$$
\int_{0}^{\infty} K_{\beta, l}(s) d s=1
$$


(see for example [20, Lemma 10.47, p. 158]). Hence, we see from (2.24) that, using the strong continuity and the dominated convergence theorem,

$$
\left\|D_{\varepsilon}^{\alpha} J_{\mu}^{\alpha} f-f\right\|_{p} \leq \int_{0}^{\infty}\left|K_{\alpha / 2, l}(s)\right| \cdot\left\|e^{-\varepsilon s} G_{\varepsilon s} f-f\right\|_{p} d s \rightarrow 0
$$

as $\varepsilon \rightarrow 0$, for any $f \in L_{p}(\mu)$ and $1 \leq p<\infty$.

Now let $p=\infty$ and $f \in L_{\infty}(\mu)$. Since $\left\|e^{-\varepsilon s} G_{\varepsilon s} f\right\|_{\infty} \leq\|f\|_{\infty}$ for $s \geq 0$, and by continuity,

$$
\lim _{\varepsilon \rightarrow 0} e^{-\varepsilon s} G_{\varepsilon s} f(x)=f(x) \quad(s>0)
$$

for $\mu$-almost all $x \in X$, we see from (2.24) that $\lim _{\varepsilon \rightarrow 0} D_{\varepsilon}^{\alpha} J_{\mu}^{\alpha} f(x)=f(x)$ for $\mu$-almost all $x \in X$ by using again the dominated convergence theorem, which proves $(2.21)$.

Remarks. 1 . Note that for $p=2$, Theorem 2.5 can simply be obtained by using the spectral resolution, since

$$
\left(J_{\mu}^{\alpha}\right)^{-1}=(I-A)^{\alpha / 2}=\frac{1}{\chi(\alpha / 2, l)} \int_{0}^{\infty} t^{-\alpha / 2-1}\left(I-e^{-t} G_{t}\right)^{l} d t .
$$

The Riesz potential operator $I_{\mu}^{\alpha}$ also has an inverse for $\alpha>0$ and $1 \leq$ $p<\infty$ (see for example [21, (5.85), p. 121]); in particular, for $p=2$ we have

$$
\begin{aligned}
\left(I_{\mu}^{\alpha}\right)^{-1}=(-A)^{\alpha / 2}=\frac{1}{\chi(\alpha / 2, l)} \int_{0}^{\infty} t^{-\alpha / 2-1}\left(I-G_{t}\right)^{l} d t & (l=[\alpha / 2]+1) .
\end{aligned}
$$

2. For $0<\alpha<2$, the formulas (2.25) and (2.26) are called the Balakrishnan formulas (see for example [28, p. 260]). Note that (2.25) or (2.26) can also be formally obtained by using the fact that

$$
(1+u)^{\alpha / 2}=\frac{1}{\chi(\alpha / 2, l)} \int_{0}^{\infty} t^{-\alpha / 2-1}\left(1-e^{-t(1+u)}\right)^{l} d t, \quad 0<\alpha / 2<l,
$$

and replacing $u$ by $-A$ or $1+u$ by $-A$.

For $\alpha>0$, let $D_{\varepsilon}^{\alpha}$ be as defined in (2.18) with $l=[\alpha / 2]+1$. Define the linear operator $D^{\alpha}$ by

$$
\lim _{\varepsilon \rightarrow 0}\left\|D_{\varepsilon}^{\alpha} f-D^{\alpha} f\right\|_{p}=0
$$

for suitable $f \in L_{p}(\mu)$ where $1 \leq p<\infty$. Then $D^{\alpha}$ can be interpreted as $\left(I-A_{p}\right)^{\alpha / 2}$.

2.3. Local heat kernels with decay conditions. We now turn back to the assumptions (G1)-(G5). In order to study the Bessel potentials $J_{\mu}^{\alpha}$ in more detail, we need more conditions on the heat kernel $G(t, x, y)$. We say that a heat kernel $G(t, x, y)$ is local, and satisfies a two-sided estimate respectively, if 
(G6) (locality): $\lim _{t \rightarrow 0} t^{-1} G(t, x, y)=0$ for any $x, y \in X(x \neq y)$,

(G7) (estimate):

$$
t^{-d / w} \Phi_{1}\left(t^{-1 / w} \varrho(x, y)\right) \leq G(t, x, y) \leq t^{-d / w} \Phi_{2}\left(t^{-1 / w} \varrho(x, y)\right)
$$

for all $x, y \in X, 0<t<r_{0}$ and some $d>0, w \geq 2$, where $r_{0}=\operatorname{diam}(X)\left(r_{0}=\infty\right.$ if $X$ is unbounded $)$, and $\Phi_{i}(i=1,2)$ are bounded, decreasing functions on $[0, \infty)$.

For $X$ bounded, as a complementary condition to (G7), we assume that

(G8) (large-time behavior): $G(t, x, y) \leq C t^{-d / w} \exp (t / 2)$ for all $x, y \in X$ and all $t>r_{0}$, where $C>0$.

Condition (G8) is very weak and can be obtained from the Nash inequality (see [6, Theorem (2.1), p. 251], by taking $\delta=1 / 2$ ) if $X$ is bounded. Under these conditions the operator $A_{p}$ is local and will be interpreted as p-Laplacian.

Clearly the Gauss-Weierstrass heat kernel $G_{\mathbb{R}^{n}}(t, x, y)$ of $(2.2)$ satisfies (G6), and (G7) with $d=n, w=2$, and

$$
\Phi_{i}(s)=(4 \pi)^{-n / 2} \exp \left(-s^{2} / 4\right) \quad(i=1,2),
$$

whilst the Cauchy-Poisson heat kernel satisfies (G7) with $d=n, w=1$, and

$$
\Phi_{i}(s)=C_{n}\left(1+s^{2}\right)^{-(n+1) / 2} \quad(s>0, i=1,2),
$$

but it is not local.

The Barlow-Bass heat kernel on the Sierpiński carpet satisfies (G7) with

$$
\Phi_{i}(s)=a_{i} \exp \left(-b_{i} s^{\gamma}\right), \quad i=1,2 .
$$

It is easy to see from (2.28) that (G6) holds, since for $x, y \in X(x \neq y)$,

$$
\begin{aligned}
0 & \leq t^{-1} G(t, x, y) \leq t^{-1-d / w} \Phi_{2}\left(t^{-1 / w} \varrho(x, y)\right) \\
& =\varrho(x, y)^{-(d+w)} s^{d+w} \Phi_{2}(s) \quad\left(s=t^{-1 / w} \varrho(x, y)\right) \\
& \rightarrow 0
\end{aligned}
$$

as $t \rightarrow 0+($ or $s \rightarrow \infty)$.

Condition (G6) may be dropped if the heat kernel $G(t, x, y)$ satisfies (G7) with

$$
\lim _{s \rightarrow \infty} s^{d+w} \Phi_{2}(s)=0 .
$$

For a stable-like process on the Sierpiński carpet in $\mathbb{R}^{n}$, the heat kernel $p^{(\sigma)}(t, x, y)$ satisfies (G7) with $w$ replaced by $\sigma w$, and

$$
\Phi_{i}(s)=a_{i}(1+s)^{-(d+\sigma w)} \quad(s \geq 0, i=1,2)
$$

(see Example 2.2). Clearly $p^{(\sigma)}(t, x, y)$ is not local. 
Condition (G7), together with

$$
\int_{0}^{\infty} s^{d-1} \Phi_{2}(s) d s<\infty,
$$

will imply that $\mu$ is a $d$-measure, that is,

$$
C^{-1} r^{d} \leq \mu(B(x, r)) \leq C r^{d}
$$

for all $x \in G$ and all $0<r<r_{0}$, where $C>0$ and $B(x, r)=\{y \in X$ : $\varrho(y, x)<r\}$ is the ball in $X$ with center $x$ and radius $r$. More precisely,

Proposition 2.6. Let $(X, \varrho, \mu)$ be a connected metric measure space endowed with a stochastically complete heat kernel $G(t, x, y)$ satisfying (G7) with (2.31). Then $\mu$ is a d-measure.

Proof. This result for $r_{0}=\infty$ (that is, $X$ unbounded) was obtained in [12, Theorem 3.2, p. 2071]. We only consider $r_{0}<\infty$. The proof is essentially the same. In fact, similar to [12, (3.3), p. 2071], we can show that there is a $C>0$ such that

$$
\mu(B(x, r)) \leq C r^{d}
$$

for all $x \in G$ and $0<r \leq c_{1}$, where $c_{1}=\min \left(r_{0}, r_{0}^{1 / w}\right)$. Without loss of generality we assume $c_{1}<r_{0}$. Noting that $\mu(X)<\infty$ since $X$ is bounded, we see that (2.33) also holds for all $c_{1}<r \leq r_{0}$, by changing the constant $C$ when necessary. Thus (2.33) holds for all $0<r \leq r_{0}$ and all $x \in X$ (in fact, (2.33) also holds for all $r_{0}<r<\infty$ since $\mu$ is supported on $X$ ).

In order to show the opposite inequality, we note that there is a small number $\varepsilon_{0} \in\left(0, r_{0}^{1-w}\right)$ such that

$$
\int_{X \backslash B(x, r)} G(t, x, y) d \mu(y) \leq \frac{1}{2}
$$

for all $x \in X$ and all $0<r \leq r_{0}$, if $0<t \leq \varepsilon_{0} r^{w}$, by using (2.31) and (2.33) (cf. [12, (3.6), p. 2072]), and so

Therefore

$$
\int_{B(x, r)} G(t, x, y) d \mu(y) \geq \frac{1}{2} .
$$

$$
\mu(B(x, r)) \geq \frac{1}{2}\left(\sup _{y \in B(x, r)} G(t, x, y)\right)^{-1}
$$

for all $x \in X, 0<r \leq r_{0}$ and all $0<t \leq \varepsilon_{0} r^{w}$. In particular, we take $t=\varepsilon_{0} r^{w}$ and then use (G7) to obtain

$$
\sup _{y \in B(x, r)} G(t, x, y) \leq \Phi_{2}(0) t^{-d / w}=C r^{-d}
$$


for all $x \in X$ and $0<r \leq r_{0}$. Thus, it follows from (2.34) that

$$
\mu(B(x, r)) \geq C r^{d}
$$

for all $x \in X$ and $0<r \leq r_{0}$.

From now on we assume that

(Main Assumption): There exists a local, stochastically complete heat kernel $G(t, x, y)$ on $(X, \varrho, \mu)$, that is, there is a function $G(t, x, y)$ on $(0, \infty) \times X \times X$ satisfying $(\mathrm{G} 1)-(\mathrm{G} 6)$.

Condition (G7) with (2.31) guarantees the existence of integral kernels of $J_{\mu}^{\alpha}$ and $I_{\mu}^{\alpha}$, with two-sided estimates.

Proposition 2.7. Assume that $G(t, x, y)$ additionally satisfies (G7)-(G8) with the integral condition $(2.31)$, and that $0<\alpha<(2 / w) d$. Then the potential operator $J_{\mu}^{\alpha}$ has an integral kernel, that is,

$$
J_{\mu}^{\alpha} f(x)=\int_{X} B_{\mu}^{\alpha}(x, y) f(y) d \mu(y) \quad(x \in X),
$$

for $f \in L_{p}(\mu)$ and $1 \leq p \leq \infty$, where

$$
B_{\mu}^{\alpha}(x, y)=\frac{1}{\Gamma(\alpha / 2)} \int_{0}^{\infty} t^{\alpha / 2-1} e^{-t} G(t, x, y) d t \quad(x, y \in X, x \neq y) .
$$

Moreover, there exists some $C>0$ such that

$$
B_{\mu}^{\alpha}(x, y) \leq C \varrho(x, y)^{-(d-\alpha w / 2)}
$$

for all $x, y \in X$, and

$$
B_{\mu}^{\alpha}(x, y) \geq C^{-1} \varrho(x, y)^{-(d-\alpha w / 2)}
$$

for all $x, y \in X$ with $0<\varrho(x, y) \leq \min \left(M_{0}, r_{0}\right)$, where $M_{0} \in(0, \infty)$ is any fixed number.

Proof. First let $X$ be bounded. For any $x, y \in X(x \neq y)$, we see from (G7) that

$$
\begin{aligned}
\int_{0}^{r_{0}} t^{\alpha / 2-1} e^{-t} G(t, x, y) d t & \leq \int_{0}^{r_{0}} t^{\alpha / 2-1} G(t, x, y) d t \\
& \leq \int_{0}^{r_{0}} t^{\alpha / 2-1-d / w} \Phi_{2}\left(t^{-1 / w} \varrho(x, y)\right) d t \\
& =w \varrho(x, y)^{-(d-\alpha w / 2)} \int_{\varrho(x, y) r_{0}^{-1 / w}}^{\infty} s^{d-\alpha w / 2-1} \Phi_{2}(s) d s \\
& \leq C \varrho(x, y)^{-(d-\alpha w / 2)}
\end{aligned}
$$


since, by using the monotonicity of $\Phi_{2}$,

$$
\begin{aligned}
\int_{\varrho(x, y) r_{0}^{-1 / w}}^{\infty} s^{d-\alpha w / 2-1} \Phi_{2}(s) d s & \leq \int_{0}^{\infty} s^{d-\alpha w / 2-1} \Phi_{2}(s) d s \\
& =\int_{0}^{1} s^{d-\alpha w / 2-1} \Phi_{2}(s) d s+\int_{1}^{\infty} s^{d-\alpha w / 2-1} \Phi_{2}(s) d s \\
& \leq \Phi_{2}(0) \int_{0}^{1} s^{d-\alpha w / 2-1} d s+\int_{1}^{\infty} s^{d-1} \Phi_{2}(s) d s<\infty .
\end{aligned}
$$

On the other hand, by (G8),

$$
\int_{r_{0}}^{\infty} t^{\alpha / 2-1} e^{-t} G(t, x, y) d t \leq C \int_{r_{0}}^{\infty} t^{\alpha / 2-1-d / w} e^{-t / 2} d t \leq C .
$$

Therefore,

$$
\begin{aligned}
\int_{0}^{\infty} t^{\alpha / 2-1} e^{-t} G(t, x, y) d t & =\int_{0}^{r_{0}} t^{\alpha / 2-1} e^{-t} G(t, x, y) d t+\int_{r_{0}}^{\infty} t^{\alpha / 2-1} e^{-t} G(t, x, y) d t \\
& \leq C\left(1+\varrho(x, y)^{-(d-\alpha w / 2)}\right) \leq C \varrho(x, y)^{-(d-\alpha w / 2)}
\end{aligned}
$$

for all $x, y \in X(x \neq y)$ since $X$ is bounded and $d>\alpha w / 2$. Thus (2.37) follows. On the other hand, by $(\mathrm{G} 7)$ and noting the fact that $\varrho(x, y) \leq r_{0}$, we have

$$
\begin{aligned}
& \int_{0}^{\infty} t^{\alpha / 2-1} e^{-t} G(t, x, y) d t \geq e^{-r_{0}} \int_{0}^{r_{0}} t^{\alpha / 2-1} G(t, x, y) d t \\
& \geq e^{-r_{0}} \int_{0}^{r_{0}} t^{\alpha / 2-1-d / w} \Phi_{1}\left(t^{-1 / w} \varrho(x, y)\right) d t \\
& =w e^{-r_{0}} \varrho(x, y)^{-(d-\alpha w / 2)} \int_{\varrho(x, y) r_{0}-1 / w}^{\infty} s^{d-\alpha w / 2-1} \Phi_{1}(s) d s \\
& \geq w e^{-r_{0}} \varrho(x, y)^{-(d-\alpha w / 2)} \int_{r_{0}^{1-1 / w}}^{\infty} s^{d-\alpha w / 2-1} \Phi_{1}(s) d s \\
& \geq C^{-1} \varrho(x, y)^{-(d-\alpha w / 2)} .
\end{aligned}
$$

Therefore (2.38) follows if $X$ is bounded. In a similar way, we can obtain (2.37) and (2.38) if $X$ is unbounded. The remaining statement follows from (2.14) and (2.1).

Assume additionally that $G(t, x, y)$ satisfies (G7) with (2.31). It is not hard to see from (2.36) and (G1)-(G5) that, for $x, y \in X(x \neq y)$ and 


$$
\begin{aligned}
& 0<\alpha<(2 / w) d \\
& \text { - } B_{\mu}^{\alpha}(x, y) \geq 0, B_{\mu}^{\alpha}(x, y)=B_{\mu}^{\alpha}(y, x) \text {. } \\
& \text { - } B_{\mu}^{\alpha_{1}+\alpha_{2}}(x, y)=\int_{X} B_{\mu}^{\alpha_{1}}(x, z) B_{\mu}^{\alpha_{2}}(z, y) d \mu(z)\left(\alpha_{1}, \alpha_{2}>0, \alpha_{1}+\alpha_{2}<\right. \\
& (2 / w) d) \text {. } \\
& \text { - } \lim _{\alpha \rightarrow 0+}\left\|J_{\mu}^{\alpha} f-f\right\|_{p}=0\left(f \in L_{p}(\mu), 1 \leq p<\infty\right), \int_{X} B_{\mu}^{\alpha}(x, y) d \mu(y)=1 \text {. }
\end{aligned}
$$

Proposition 2.8. Let $X$ be unbounded. Assume that $G(t, x, y)$ additionally satisfies (G7) with the integral condition (2.31), and that $0<\alpha$ $<(2 / w) d$. Then the potential operator $I_{\mu}^{\alpha}$ has an integral kernel, that is,

$$
I_{\mu}^{\alpha} f(x)=\int_{X} R_{\mu}^{\alpha}(x, y) f(y) d \mu(y)
$$

with

$$
R_{\mu}^{\alpha}(x, y)=\frac{1}{\Gamma(\alpha / 2)} \int_{0}^{\infty} t^{\alpha / 2-1} G(t, x, y) d t \quad(x, y \in X, x \neq y) .
$$

Moreover,

$$
R_{\mu}^{\alpha}(x, y) \cong \varrho(x, y)^{-(d-\alpha w / 2)} \quad(x, y \in X, x \neq y) .
$$

Proof. The proof is similar to that of Proposition 2.7. We omit the details.

Definition 2.9. Assume that $G(t, x, y)$ satisfies (G1)-(G7) on a measure metric space $(X, \varrho, \mu)$ with the chain condition. For $\alpha>0$ and $1 \leq p \leq \infty$, the $J_{\mu}^{\alpha}$ and $I_{\mu}^{\alpha}$ defined as in (2.14) and (2.15) are termed the $p$-Bessel and p-Riesz potential operators respectively.

The operators $D^{\alpha}=(I-A)^{\alpha / 2}$ and $(-A)^{\alpha / 2}$ may be interpreted as Bessel and Riesz fractional derivatives, respectively.

3. Sobolev spaces. Assume that $G(t, x, y)$ satisfies (G1)-(G7). For each $\alpha>0$, let $J_{\mu}^{\alpha}$ be the Bessel potential operator defined in (2.14). In this section we introduce (fractional) Sobolev-type spaces on $(X, \varrho, \mu)$. For $0<\alpha \leq 1$ and $p=2$, these spaces are shown to coincide with Lipschitz spaces initiated by Jonsson and Wallin $[16,17]$. If $X$ is an open subset of $\mathbb{R}^{n}$ with "nice" boundary, they are equivalent to the usual classical Sobolev spaces. These function spaces arise as the domains of $\left(I-A_{p}\right)^{\alpha / 2}$, and play an important rôle in studying nonlinear (fractional) PDE's on $(X, \varrho, \mu)$. Their embedding theorems will be given in the next section. Note that Theorem 2.5 is crucial to our argument.

For any $\alpha>0$ and $1 \leq p \leq \infty$, we see from (2.20) and (2.21) that the Bessel potential operator $J_{\mu}^{\alpha}: L_{p}(\mu) \rightarrow L_{p}(\mu)$ is one-to-one. 
Definition 3.1. Let $\alpha>0$ and $1 \leq p \leq \infty$. The Sobolev space (or Bessel potential space) $H_{p}^{\alpha}(\mu):=H_{p}^{\alpha}(X, \varrho, \mu, G)$ on $(X, \varrho, \mu)$ is the image of $L_{p}(\mu)$ under $J_{\mu}^{\alpha}$. The norm of $f=J_{\mu}^{\alpha} \varphi \in H_{p}^{\alpha}(\mu)$ is

$$
\|f\|_{H_{p}^{\alpha}(\mu)}=\|\varphi\|_{p} .
$$

Strichartz [23] introduced the Sobolev-type spaces $L_{s}^{p}(X)$ for $s>0$ and $1<p<\infty$. It is easy to see that

$$
L_{s}^{p}(X)=H_{p}^{2 s / w}(\mu) \quad(s>0,1<p<\infty) .
$$

We may characterize $H_{p}^{\alpha}(\mu)$ in an alternative way. Let $\alpha>0$ and $1 \leq p<\infty$. Define the space $L_{p, p}^{\alpha}(\mu)$ by

$$
L_{p, p}^{\alpha}(\mu)=\left\{f \in L_{p}(\mu): D^{\alpha} f \in L_{p}(\mu)\right\},
$$

where $D^{\alpha} f$ is defined in (2.27). The norm of $f \in L_{p, p}^{\alpha}(\mu)$ is

$$
\|f\|_{L_{p, p}^{\alpha}(\mu)}=\|f\|_{p}+\left\|D^{\alpha} f\right\|_{p} .
$$

Proposition 3.2. Let $\alpha>0$ and $1 \leq p<\infty$. Then $H_{p}^{\alpha}(\mu)=L_{p, p}^{\alpha}(\mu)$ with equivalent norms:

$$
\|f\|_{H_{p}^{\alpha}(\mu)} \leq\|f\|_{L_{p, p}^{\alpha}(\mu)} \leq 2\|f\|_{H_{p}^{\alpha}(\mu)} .
$$

Proof. Assume that $f \in H_{p}^{\alpha}(\mu)$. Write $f=J_{\mu}^{\alpha} \varphi$ for some $\varphi \in L_{p}(\mu)$. It follows from (2.20) and (2.27) that

$$
D^{\alpha} f=\lim _{\varepsilon \rightarrow 0} D_{\varepsilon}^{\alpha} f=\lim _{\varepsilon \rightarrow 0} D_{\varepsilon}^{\alpha} J_{\mu}^{\alpha} \varphi=\varphi
$$

in the $L_{p}$-norm since $1 \leq p<\infty$. Thus,

$$
\|f\|_{p}+\left\|D^{\alpha} f\right\|_{p}=\left\|J_{\mu}^{\alpha} \varphi\right\|_{p}+\|\varphi\|_{p} \leq 2\|\varphi\|_{p}=2\|f\|_{H_{p}^{\alpha}(\mu)},
$$

proving that $H_{p}^{\alpha}(\mu)$ is embedded in $L_{p, p}^{\alpha}(\mu)$. Conversely, assume that $f \in$ $L_{p, p}^{\alpha}(\mu)$. Let $\varphi=D^{\alpha} f \in L_{p}(\mu)$. Then $f=J_{\mu}^{\alpha} \varphi$; this is because, for any $g \in L^{p^{\prime}}(\mu)\left(p^{\prime}\right.$ is the conjugate of $\left.p\right)$ we have

$$
\left(J_{\mu}^{\alpha} \varphi, g\right)=\left(\varphi, J_{\mu}^{\alpha} g\right)=\lim _{\varepsilon \rightarrow 0}\left(D_{\varepsilon}^{\alpha} f, J_{\mu}^{\alpha} g\right)=\lim _{\varepsilon \rightarrow 0}\left(f, D_{\varepsilon}^{\alpha} J_{\mu}^{\alpha} g\right)=(f, g) .
$$

(Note that the last equality still holds if $p=1$ by using (2.21) and the dominated convergence theorem.) Therefore, we see that $f=J_{\mu}^{\alpha} \varphi \in H_{p}^{\alpha}(\mu)$, and

$$
\|f\|_{H_{p}^{\alpha}(\mu)}=\|\varphi\|_{p}=\left\|D^{\alpha} f\right\|_{p} \leq\|f\|_{L_{p, p}^{\alpha}(\mu)}
$$

showing that $L_{p, p}^{\alpha}(\mu)$ is embedded in $H_{p}^{\alpha}(\mu)$.

In what follows we consider the case $p=2$, and investigate $H_{2}^{\alpha}(\mu)$ in more detail. Let $\alpha>1$ and $f \in H_{2}^{\alpha}(\mu)$. We claim that $(-A)^{1 / 2} f \in H_{2}^{\alpha-1}(\mu)$. In fact, writing $f=J_{\mu}^{\alpha} \varphi$ for some $\varphi \in L_{2}(\mu)$, we see from Proposition 2.4 that

$$
(-A)^{1 / 2} f=(I-A)^{-(\alpha-1) / 2} \widetilde{\varphi}
$$


where $\widetilde{\varphi}=(I-A)^{-1 / 2}(-A)^{1 / 2} \varphi$, by using the operational calculus (cf. [28, pp. 343-345]). Note that $\widetilde{\varphi} \in L_{2}(\mu)$ for $\varphi \in L_{2}(\mu)$. Thus $(-A)^{1 / 2} f=$ $J_{\mu}^{\alpha-1} \widetilde{\varphi} \in H_{2}^{\alpha-1}(\mu)$. Conversely, if $(-A)^{1 / 2} f \in H_{2}^{\alpha-1}(\mu)(\alpha>1)$ then $f \in$ $H_{2}^{\alpha}(\mu)$ in a similar way. The Riesz fractional derivative $(-A)^{1 / 2}$ behaves like a pseudo-differential operator of order 1 , exactly the same as the classical case. On the other hand, if we instead consider $L_{s}^{p}(X)$ introduced by Strichartz as above, then we see that $(-A)^{1 / 2} f \in L_{s-w / 2}^{2}(X)(s>w / 2)$ if and only if $f \in L_{s}^{2}(X)$, and $(-A)^{1 / 2}$ behaves like a pseudo-differential operator of order $w / 2$.

Using the spectral resolution (cf. Proposition 2.4), we see that for any $\alpha>0$,

$$
H_{2}^{\alpha}(\mu)=\left\{f \in L_{2}(\mu): \int_{0}^{\infty}(1+\lambda)^{\alpha} d\left(E_{\lambda} f, f\right)<\infty\right\} .
$$

For $0<\alpha \leq 1$, we give a simple characterization of $H_{2}^{\alpha}(\mu)$. To do this, we introduce a function $j_{\alpha}: X \times X \rightarrow \mathbb{R}$ for $\alpha>0$ by $j_{\alpha}(x, y)=0$ if $x=y$, and

$$
j_{\alpha}(x, y)=\frac{1}{\chi(\alpha, 1)} \int_{0}^{\infty} t^{-\alpha-1} e^{-t} G(t, x, y) d t
$$

if $x \neq y$. Define a functional $W_{\alpha}$ by

$$
W_{\alpha}(f)=\int_{X} \int_{X}(f(x)-f(y))^{2} j_{\alpha}(x, y) d \mu(y) d \mu(x)
$$

for $f \in L_{2}(\mu)$.

Theorem 3.3. Let $H_{2}^{\alpha}(\mu)$ be defined as above. Then

$$
H_{2}^{\alpha}(\mu)=\left\{f \in L_{2}(\mu): W_{\alpha}(f)<\infty\right\}
$$

with $\|f\|_{H_{2}^{\alpha}(\mu)} \cong\left(\|f\|_{2}^{2}+W_{\alpha}(f)\right)^{1 / 2}$ if $0<\alpha<1$, while

$$
H_{2}^{1}(\mu)=\left\{f \in L_{2}(\mu): \mathcal{E}(f, f)<\infty\right\}
$$

with $\|f\|_{H_{2}^{1}(\mu)} \cong\left(\|f\|_{2}^{2}+\mathcal{E}(f, f)\right)^{1 / 2}$, where $\mathcal{E}$ is defined in (2.13).

Proof. Let $0<\alpha<1$. Let $f \in H_{2}^{\alpha}(\mu)$, and write $f=J_{\mu}^{\alpha} \varphi$ for some $\varphi \in L_{2}(\mu)$. Then

$$
\varphi=D^{\alpha} f=(I-A)^{\alpha / 2} f .
$$

Therefore,

$$
\begin{aligned}
\|f\|_{H_{2}^{\alpha}(\mu)}^{2} & =\|\varphi\|_{2}^{2}=\left((I-A)^{\alpha / 2} f,(I-A)^{\alpha / 2} f\right)=\left(f,(I-A)^{\alpha} f\right) \\
& =\left(f, D^{2 \alpha} f\right)=\frac{1}{\chi(\alpha, 1)} \int_{0}^{\infty} t^{-\alpha-1}\left(f, f-e^{-t} G_{t} f\right) d t
\end{aligned}
$$




$$
\begin{aligned}
& =\frac{1}{\chi(\alpha, 1)} \int_{0}^{\infty} t^{-\alpha-1}\left[\left(1-e^{-t}\right)\|f\|_{2}^{2}+e^{-t}\left(f, f-G_{t} f\right)\right] d t \\
& =\|f\|_{2}^{2}+\frac{1}{2 \chi(\alpha, 1)} \int_{0}^{\infty} t^{-\alpha-1} e^{-t}\left[\int_{X} \int_{X}(f(y)-f(x))^{2} G(t, x, y) d \mu(y) d \mu(x)\right] d t \\
& =\|f\|_{2}^{2}+\frac{1}{2} \int_{X} \int_{X}(f(y)-f(x))^{2} j_{\alpha}(x, y) d \mu(y) d \mu(x),
\end{aligned}
$$

proving (3.7). Moreover,

$$
\begin{aligned}
\|f\|_{H_{2}^{1}(\mu)}^{2} & =\|\varphi\|_{2}^{2}=\left((I-A)^{1 / 2} f,(I-A)^{1 / 2} f\right) \\
& =\int_{0}^{\infty}(1+\lambda) d\left(E_{\lambda} f, f\right)=\|f\|_{2}^{2}+\mathcal{E}(f, f),
\end{aligned}
$$

and so (3.8) holds.

Corollary 3.4. Suppose that $(X, \varrho, \mu)$ is a connected metric measure space, endowed with a local, stochastically complete heat kernel $G(t, x, y)$ satisfying (G7) with

$$
\int_{0}^{\infty} s^{d+w-1} \Phi_{2}(s) d s<\infty .
$$

Then $H_{2}^{\alpha}(\mu)=H_{2}^{\alpha}(d, w)$ with equivalent norms, where

$$
\begin{aligned}
H_{2}^{\alpha}(d, w)= & \left\{f \in L_{2}(\mu):\right. \\
& \left.\int_{X} \int_{\varrho(y, x) \leq 1} \frac{(f(y)-f(x))^{2}}{\varrho(x, y)^{d+\alpha w}} d \mu(y) d \mu(x)<\infty\right\}
\end{aligned}
$$

if $0<\alpha<1$, whilst

$$
\begin{aligned}
H_{2}^{1}(d, w)= & \left\{f \in L_{2}(\mu):\right. \\
& \left.\sup _{0<r<1} r^{-(d+w)} \int_{X} \int_{B(x, r)}(f(y)-f(x))^{2} d \mu(y) d \mu(x)<\infty\right\}
\end{aligned}
$$

The norm of $f \in H_{2}^{\alpha}(d, w)$ is defined in an obvious way for $\alpha>0$.

REMARK. Note that under the conditions of Corollary 3.4, the space $H_{2}^{\alpha}(d, w)$ defined in (3.10) contains only constant functions if $\alpha \geq 1$ (see [19]).

Proof. Assume $0<\alpha<1$. Since $G(t, x, y)$ satisfies (G7) with (3.9), we can obtain $j_{\alpha}(x, y) \leq C \varrho(x, y)^{-(d+\alpha w)}$ for $x, y \in X(x \neq y)$, and

$$
j_{\alpha}(x, y) \geq C^{-1} \varrho(x, y)^{-(d+\alpha w)}
$$

if $0<\varrho(x, y) \leq 1$, in a similar way to (2.37), whether $X$ is bounded or not, where $C$ is independent of $x, y$. Thus (3.10) follows from (3.7). For $\alpha=1$, 
we have

$$
\mathcal{E}(f, f) \cong \sup _{0<r<1} r^{-(d+w)} \int_{X} \int_{B(x, r)}(f(y)-f(x))^{2} d \mu(y) d \mu(x)
$$

by virtue of (G7) and (3.9) (see the details in [12, Theorem 4.2, p. 2076]; note that $\mu$ is a $d$-measure if (G7) holds with (3.9), see Proposition 2.6). Thus (3.11) follows from (3.8).

REMARK. If $X$ is a subset of $\mathbb{R}^{n}$ supporting a $d$-measure, the spaces $H_{2}^{\alpha}(d, w)$ for $0<\alpha \leq 1$ and $w=2$ were introduced by Jonsson and Wallin in [16, 17]; in particular, they are the traces of the Sobolev spaces $H_{2}^{\alpha+(n-d) / 2}\left(\mathbb{R}^{n}\right)$ on $X$ for $0<\alpha<1$.

4. Embedding theorems. Assume that $G(t, x, y)$ satisfies (G1)-(G7) with (3.9). In this section we discuss the embedding theorems for $H_{p}^{\alpha}(\mu)$ for $\alpha>0$ and $1<p<\infty$.

Theorem 4.1. Let $H_{p}^{\alpha}(\mu)$ be defined as in Definition 3.1 for $\alpha>0$ and $1<p<\infty$. Then

(1) if $d>\alpha p w / 2$, then $H_{p}^{\alpha}(\mu)$ embeds in $L^{q}(\mu)$ for $q=\frac{p d}{d-\alpha p w / 2}$;

(2) if $d=\alpha p w / 2$, then $H_{p}^{\alpha}(\mu)$ embeds in $L^{q}(\mu)$ for any $1<q<\infty$.

Proof. (1) Assume $d>\alpha p w / 2$. Let $f \in H_{p}^{\alpha}(\mu)$, and write $f=J_{\mu}^{\alpha} \varphi$, $\varphi \in L_{p}(\mu)$. Since $0<\alpha<2 d / p w<(2 / w) d$, we see from (2.37) that there exists some $C>0$ independent of $x$ and $f$ such that

$$
|f(x)| \leq C \int_{X} \varrho(x, y)^{-(d-\alpha w / 2)}|\varphi(y)| d \mu(y),
$$

which implies that $f \in L^{q}(\mu)$ if $d>\alpha p w / 2$. The proof for this is standard (see for example [14, pp. 20-21]). For the reader's convenience, we sketch the arguments. Write

$$
\int_{X} \varrho(x, y)^{-(d-\alpha w / 2)}|\varphi(y)| d \mu(y)
$$

$$
\begin{aligned}
& =\int_{\varrho(y, x)>\delta} \varrho(x, y)^{-(d-\alpha w / 2)}|\varphi(y)| d \mu(y)+\int_{\varrho(y, x) \leq \delta} \varrho(x, y)^{-(d-\alpha w / 2)}|\varphi(y)| d \mu(y) \\
& =: g_{\delta}(x)+b_{\delta}(x) .
\end{aligned}
$$

Using Hölder's inequality, we have

$$
\begin{aligned}
g_{\delta}(x) & \leq\|\varphi\|_{p}\left(\int_{\varrho(x, y)>\delta} \varrho(x, y)^{-p^{\prime}(d-\alpha w / 2)} d \mu(y)\right)^{1 / p^{\prime}} \\
& \leq C\|\varphi\|_{p} \delta^{-(d / p-\alpha w / 2)}
\end{aligned}
$$


where $C$ is independent of $\varphi, \delta$ and $x$, and $p^{\prime}=p /(p-1)$. Here we have used the fact that

$$
\begin{aligned}
\int_{\varrho(x, y)>\delta} \varrho(x, y)^{-p^{\prime}(d-\alpha w / 2)} & d \mu(y)=\sum_{k=1}^{\infty} \int_{2^{k} \delta<\varrho(x, y) \leq 2^{k+1} \delta} \varrho(x, y)^{-p^{\prime}(d-\alpha w / 2)} d \mu(y) \\
& \leq \sum_{k=1}^{\infty}\left(2^{k} \delta\right)^{-p^{\prime}(d-\alpha w / 2)} \mu\left(B\left(x, 2^{k+1} \delta\right)\right) \\
& \leq \sum_{k=1}^{\infty}\left(2^{k} \delta\right)^{-p^{\prime}(d-\alpha w / 2)} C\left(2^{k+1} \delta\right)^{d} \quad(\text { by }(2.33)) \\
& =C \delta^{d-p^{\prime}(d-\alpha w / 2)} \sum_{k=1}^{\infty} 2^{k\left(d-p^{\prime}(d-\alpha w / 2)\right)} \\
& \leq C \delta^{d-p^{\prime}(d-\alpha w / 2)}\left(\text { since } d-p^{\prime}(d-\alpha w / 2)<0\right) .
\end{aligned}
$$

On the other hand,

$$
\begin{aligned}
b_{\delta}(x) & =\int_{\varrho(x, y) \leq \delta} \varrho(x, y)^{-(d-\alpha w / 2)}|\varphi(y)| d \mu(y) \\
& =\sum_{k=0}^{\infty} \int_{2^{-(k+1)}} \int_{\delta<\varrho(x, y) \leq 2^{-k} \delta} \varrho(x, y)^{-(d-\alpha w / 2)}|\varphi(y)| d \mu(y) \\
& \leq \sum_{k=0}^{\infty}\left(2^{-(k+1)} \delta\right)^{-(d-\alpha w / 2)} \int_{\varrho(x, y) \leq 2^{-k} \delta}|\varphi(y)| d \mu(y) \\
& \leq \sum_{k=0}^{\infty}\left(2^{-(k+1)} \delta\right)^{-(d-\alpha w / 2)} \mu\left(B\left(x, 2^{-k} \delta\right)\right) M_{\mu}(\varphi)(x) \\
& \leq C \delta^{\alpha w / 2} M_{\mu}(\varphi)(x),
\end{aligned}
$$

where

$$
M_{\mu} \varphi(x):=\sup _{0<r \leq r_{0}} \frac{1}{\mu(B(x, r))} \int_{B(x, r)}|\varphi(y)| d \mu(y)
$$

is the maximal function of $\varphi$ satisfying

$$
\left\|M_{\mu} \varphi\right\|_{p} \leq C\|\varphi\|_{p} \quad \text { for all } \varphi \in L_{p}(\mu) .
$$

Combining (4.1)-(4.5), we see that

$$
|f(x)| \leq C\left(\delta^{-(d / p-\alpha w / 2)}\|\varphi\|_{p}+\delta^{\alpha w / 2} M_{\mu} \varphi(x)\right) .
$$

Minimizing the right-hand side, we have

$$
|f(x)| \leq C\|\varphi\|_{p}^{\alpha w p / 2 d}\left(M_{\mu} \varphi(x)\right)^{(2 d-\alpha w p) / 2 d},
$$


which combines with (4.5) to yield

$$
\|f\|_{q} \leq C\|\varphi\|_{p}, \quad q=\frac{d p}{d-\alpha p w / 2} .
$$

Case (2), $d=\alpha p w / 2$, follows from (1) in a standard way. We omit the details.

We give the embedding of $H_{p}^{\alpha}(\mu)$ for $p=2$, if $d<\alpha w$ and $0<\alpha \leq 1$. For $0<\sigma \leq 1$, let $C^{\sigma}(X)$ be the Hölder space on $X$, that is,

$$
C^{\sigma}(X)=\left\{f \in C(X): \sup _{x, y \in X, x \neq y} \frac{|f(y)-f(x)|}{\varrho(y, x)^{\sigma}}<\infty\right\} .
$$

The norm of $f \in C^{\sigma}(X)$ is

$$
\|f\|_{C^{\sigma}(X)}=\|f\|_{C(X)}+\sup _{x, y \in X, x \neq y} \frac{|f(y)-f(x)|}{\varrho(y, x)^{\sigma}} .
$$

Theorem 4.2. If $d<\alpha w$ and $0<\alpha \leq 1$, then $H_{2}^{\alpha}(\mu)$ embeds in $C^{\sigma}(X)$, where $\sigma=(\alpha w-d) / 2$.

Proof. Let $d<\alpha w$ and $0<\alpha \leq 1$. By Corollary 3.4, it suffices to show that $H_{2}^{\alpha}(d, w)$ embeds in $C^{\sigma}(X)$ with $\sigma=(\alpha w-d) / 2$. But this is proved in Theorem 4.1(iii) of [12] if $\alpha=1$, and in [15] for $0<\alpha<1$. (Note that the chain condition implies that $\alpha w \leq d+2$ cf. [12], and so $\sigma \leq 1$.)

By (3.2) and Theorem 4.2 we infer that $L_{s}^{2}(X)$ embeds in $C^{\sigma}(X)$ with $\sigma=s-d / 2$ if $d / 2<s \leq w / 2$ (see the same result in [23]).

5. Besov spaces. Let $(X, \varrho, \mu)$ be a connected metric measure space endowed with a $G(t, x, y)$ satisfying $(\mathrm{G} 1)-(\mathrm{G} 7)$. Let $\left\{G_{t}\right\}_{t \geq 0}$ be the semigroup associated with $G(t, x, y)$ as in (2.1). In this section we define various Besov spaces $B_{p, q}^{\alpha}(\mu)$ for $\alpha \in \mathbb{R}$ and $1 \leq p, q \leq \infty$. follows:

Definition 5.1. Let $1 \leq p, q \leq \infty$, and let $\alpha \in \mathbb{R}$. Define $B_{p, q}^{\alpha}(\mu)$ as

(1) if $\alpha \geq 0, k=[\alpha / 2]+1$, then

$$
B_{p, q}^{\alpha}(\mu)=\left\{f \in L_{p}(\mu):\left(\int_{0}^{\infty}\left(t^{k-\alpha / 2}\left\|\frac{\partial^{k}}{\partial t^{k}} G_{t} f\right\|_{p}\right)^{q} \frac{d t}{t}\right)^{1 / q}<\infty\right\} ;
$$

(2) if $\alpha<0$, take $k=0$ :

$$
B_{p, q}^{\alpha}(\mu)=\left\{f \in L_{p}(\mu):\left(\int_{0}^{\infty}\left(t^{-\alpha / 2}\left\|G_{t} f\right\|_{p}\right)^{q} \frac{d t}{t}\right)^{1 / q}<\infty\right\}
$$

with the obvious norm (the integrals above are clearly modified if $q=\infty$ ). 
The Besov spaces as above for the classical case were given in [10] by using the Gauss-Weierstrass heat kernel. For other approaches to the fractal case, see $[8,23,24,26]$.

Theorem 5.2. For $\alpha \geq 0$, we have $B_{2,2}^{\alpha}(\mu)=H_{2}^{\alpha}(\mu)$ with equivalent norms.

Proof. Note that

$$
H_{2}^{\alpha}(\mu)=\left\{f \in L_{2}(\mu): \int_{0}^{\infty}(1+\lambda)^{\alpha} d\left(E_{\lambda} f, f\right)<\infty\right\} \quad(\alpha \geq 0) .
$$

On the other hand, for any integer $k \geq[\alpha / 2]+1$, we have

$$
\left\|\frac{\partial^{k}}{\partial t^{k}} G_{t} f\right\|_{2}^{2}=\int_{0}^{\infty} \lambda^{2 k} e^{-2 \lambda t} d\left(E_{\lambda} f, f\right)
$$

and so

$$
\begin{aligned}
\int_{0}^{\infty} t^{2 k-\alpha-1}\left\|\frac{\partial}{\partial t} G_{t} f\right\|_{2}^{2} d t & =\int_{0}^{\infty} t^{2 k-\alpha-1}\left(\int_{0}^{\infty} \lambda^{2 k} e^{-2 \lambda t} d\left(E_{\lambda} f, f\right)\right) d t \\
& =\int_{0}^{\infty} \lambda^{2 k} d\left(E_{\lambda} f, f\right)\left(\int_{0}^{\infty} t^{2 k-\alpha-1} e^{-2 \lambda t} d t\right) \\
& =2^{\alpha-2 k} \Gamma(2 k-\alpha) \int_{0}^{\infty} \lambda^{\alpha} d\left(E_{\lambda} f, f\right)
\end{aligned}
$$

proving the theorem.

6. Discussions. Starting from the existence of a local, stochastically complete heat kernel $G(t, x, y)$ with a two-sided estimate $(\mathrm{G} 7)$ on a connected metric measure space $(X, \varrho, \mu)$, we have obtained various Besov spaces; in particular, we have at hand the (fractional) Sobolev spaces $H_{2}^{\alpha}(d, w)(0<\alpha \leq 1)$ (see (3.10) and (3.11)). These spaces contain two parameters, $d$ and $w$, which are the Hausdorff dimension of $X$ and the walk dimension of the diffusion on $(X, \varrho, \mu)$ respectively. The key point is that these spaces are dense in $L_{2}(\mu)$. There is a natural question: is it possible to obtain the existence of a heat kernel satisfying a two-sided estimate (G7) if there exists a function space, say $H_{2}^{\alpha}(d, w)$ (cf. (3.10) or (3.11)) for some $0<\alpha \leq 1$, that is dense in $L_{2}(\mu)$ ? This question has been answered for $0<\alpha<1$ and $w=2$. Chen and Kumagai [7] applied the probability approach to show that, if $X$ is a $d$-subset of $\mathbb{R}^{n}(n \geq 1)$ and $\mu$ is a $d$-measure, then there is a heat kernel $p^{(\sigma)}(t, x, y)$ satisfying

$$
C^{-1} t^{-d / 2 \alpha} \Phi\left(t^{-1 / 2 \alpha}|x-y|\right) \leq p^{(\sigma)}(t, x, y) \leq C t^{-d / 2 \alpha} \Phi\left(t^{-1 / 2 \alpha}|x-y|\right)
$$


for all $0<t<1$ and all $x, y \in X$, where $0<\alpha<1$ and

$$
\Phi(s)=(1+s)^{-(d+2 \alpha)} \quad(s \geq 0) .
$$

(Note that the problem of the denseness of $H_{2}^{\alpha}(d, w)$ does not appear if $0<\alpha \leq 1$ and $w=2$.) For each $0<\alpha<1$, the $p^{(\sigma)}(t, x, y)$ is not local, and the corresponding process is a jump process on $X$. The limiting case $\alpha=1$ is still open. Recently, in [29] a regular local Dirichlet form has been constructed on any bounded $d$-set which corresponds to the limiting case $\alpha=1$. All the results mentioned above deal only with $w=2$. It would be interesting to investigate the general case $w>2$, and moreover, to show the equivalence between the existence of a certain class of potential spaces and the existence of heat kernels with two-sided decay estimates.

\section{References}

[1] I. A. Aliev and S. Uyhan-Bayrakci, On inversion of Bessel potentials associated with the Laplace-Bessel differential operator, Acta Math. Hungar. 95 (2002), 125-145.

[2] M. T. Barlow, Diffusions on fractals, in: Lectures on Probability Theory and Statistics, Lecture Notes in Math. 1690, Springer, 1998, 1-121.

[3] M. T. Barlow and R. F. Bass, Brownian motion and harmonic analysis on Sierpiński carpets, Canad. J. Math. 51 (1999), 673-744.

[4] M. T. Barlow and E. A. Perkins, Brownian motion on the Sierpinski gasket, Probab. Theory Related Fields 79 (1988), 543-623.

[5] K. Bogdan, A. Stós and P. Sztonyk, Harnack inequality for stable processes on $d$-sets, Studia Math. 158 (2003), 163-198.

[6] E. A. Carlen, S. Kusuoka and D. W. Stroock, Upper bounds for symmetric Markov transition functions, Ann. Inst. H. Poincaré Probab. Statist. 23 (1987), 245-287.

[7] Z. Q. Chen and T. Kumagai, Heat kernel estimates for stable-like processes on d-sets, Stochastic Process. Appl. 108 (2003), 27-62.

[8] T. Coulhon, Off-diagonal heat kernel lower bounds without Poincaré, J. London Math. Soc. 68 (2003), 795-816.

[9] P. J. Fitzsimmons, B. M. Hambly and T. Kumagai, Transition density estimates for Brownian motion on affine nested fractals, Comm. Math. Phys. 165 (1994), 595-620.

[10] T. M. Flett, Temperatures, Bessel potentials and Lipschitz spaces, Proc. London Math. Soc. 22 (1971), 385-451.

[11] M. Fukushima, Y. Oshima and M. Takeda, Dirichlet Forms and Symmetric Markov Processes, de Gruyter, Berlin, 1994.

[12] A. Grigoryan, J. Hu and K.-S. Lau, Heat kernels on metric-measure spaces and an application to semilinear elliptic equations, Trans. Amer. Math. Soc. 355 (2003), 2065-2095.

[13] B. M. Hambly and T. Kumagai, Transition density estimates for diffusion processes on post critically finite self-similar fractals, Proc. London Math. Soc. 78 (1999), 431-458.

[14] J. Heinonen, Lectures on Analysis on Metric Spaces, Springer, 2001.

[15] J. Hu and M. Zähle, Jump processes and nonlinear fractional heat equations on fractals, Math. Nachr., to appear. 
[16] A. Jonsson, Brownian motion on fractals and function spaces, Math. Z. 222 (1996), $495-504$.

[17] A. Jonsson and H. Wallin, Function Spaces on Subsets of $\mathbb{R}^{n}$, Harwood, 1984.

[18] T. Kumagai and K. T. Sturm, Construction of diffusion processes on fractals, d-sets, and general metric measure spaces, J. Math. Kyoto Univ., to appear.

[19] K. Pietruska-Pałuba, Heat kernels on metric spaces and a characterisation of constant functions, Manuscripta Math. 115 (2004), 389-399.

[20] B. Rubin, Fractional Integrals and Potentials, Pitman Monogr. Surveys Pure Appl. Math. 82, Longman, Harlow, 1996.

[21] S. G. Samko, A. A. Kilbas and O. L. Marichev, Fractional Integrals and Derivatives, Gordon and Breach, Amsterdam, 1993.

[22] E. M. Stein, Singular Integrals and Differentiability Properties of Functions, Princeton Univ. Press, 1970.

[23] R. S. Strichartz, Function spaces on fractals, J. Funct. Anal. 198 (2003), 43-83.

[24] -, Analysis on products of fractals, Trans. Amer. Math. Soc. 357 (2005), 571-615.

[25] M. H. Taibleson, On the theory of Lipschitz spaces of distributions on Euclidean n-spaces. Principal properties, J. Math. Mech. 13 (1964), 407-479.

[26] H. Triebel, Theory of Function Spaces II, Monogr. Math. 84, Birkhäuser, Basel, 1992.

[27] —, Fractals and Spectra: Related to Fourier Analysis and Function Spaces, Birkhäuser, Basel, 1997.

[28] K. Yosida, Functional Analysis, Springer, 1980.

[29] M. Zähle, Local structures and diffusions on fractals, preprint.

Department of Mathematical Sciences

Tsinghua University

Beijing 100084, China

E-mail: hujiaxin@mail.tsinghua.edu.cn
Mathematical Institute

University of Jena

07743 Jena, Germany E-mail: zaehle@minet.uni-jena.de

Received July 20, 2004

Revised version April 13, 2005 